\title{
IGM Heating and AGN activity in Fossil Galaxy Groups
}

\author{
H. Miraghaei ${ }^{1,2}$, H. G. Khosroshahi ${ }^{2}$, H.-R. Klöckner ${ }^{3,4}$, \\ T. J. Ponman ${ }^{5}$, N. N. Jetha ${ }^{6}$, and S. Raychaudhury ${ }^{5}$ \\ ${ }^{1}$ Department of Physics, Sharif University of Technology PO Box 11365-9161, Tehran, Iran \\ email: miraghaee@physics.sharif .edu \\ ${ }^{2}$ School of Astronomy, Institute for Research in Fundamental Sciences, PO Box 19395-5531, \\ Tehran, Iran \\ ${ }^{3}$ Subdepartment of Astrophysics, University of Oxford, Denys-Wilkinson Building, Keble \\ Road, Oxford, OX1 3RH, UK \\ ${ }^{4}$ Max-Planck-Institut für Radioastronomie, Auf dem Hügel 69, 53121 Bonn, Germany \\ ${ }^{5}$ School of Physics and Astronomy, University of Birmingham, Edgbaston, Birmingham B15 \\ $2 \mathrm{TT}, \mathrm{UK}$ \\ ${ }^{6}$ Center for Space Plasma and Aeronomic Research (CSPAR), University of Alabama in \\ Huntsville, Huntsville, AL 35805, USA
}

\begin{abstract}
Fossil galaxy groups are energetically and morphologically ideal environments to study the intergalactic medium (IGM) heating, because their inter-galactic gas is undisturbed due to the lack of recent group scale mergers. We study the role of active galactic nuclei (AGN) in heating the IGM in a sample of five fossil galaxy groups by employing properties at $610 \mathrm{MHz}$ and 1.4 GHz. We find that two of the dominant galaxies in fossil groups, ESO 3060170 and RX $\mathrm{J} 1416.4+2315$, are associated with the radio lobes. We evaluate the PdV work of the radio lobes and their corresponding heating power and compare to the X-ray emission loss within cooling radius. Our results show that the power due to mechanical heating is not sufficiently high to suppress the cooling.
\end{abstract}

Keywords. galaxies: active, galaxies: clusters: general, intergalactic medium, cooling flows, radio continuum: galaxies

\section{Introduction}

Loss of energy through X-ray emissions in galaxy cluster cools down the inter-galactic gas produces a sharp surface brightness pick and temperature drop at the core (Peterson $\&$ Fabian 2006). The X-ray observations of galaxy clusters by Chandra and XMM-Newton show no sign of gas below $\sim k e V$. Among different heating mechanism proposed up to now, AGNs are introduced as the main and the most promising heating sources (Gitti et al. 2012). Due to the complex nature of the galaxy groups, which are subject to both group scale mergers and galaxy-galaxy mergers, the share of the AGN in the IGM heating can not be estimated accurately. For this study, we observed a sample of wellknown fossil groups with prior high-quality X-ray observations with the Giant Metrewave Radio Telescope (GMRT) at $610 \mathrm{MHz}$ and $1.4 \mathrm{GHz}$. A fossil group is dominated by a giant elliptical galaxy at the centriod of group X-ray emission. Their extended luminous $\mathrm{X}$-ray emission is regular and symmetric indicating the absence of recent group scale mergers (Jones et al. 2003). The main goal is the study of intergalactic medium heating in fossil galaxy groups, giving prominence to AGNs heating powers via the detection of radio bubbles at low frequencies. 


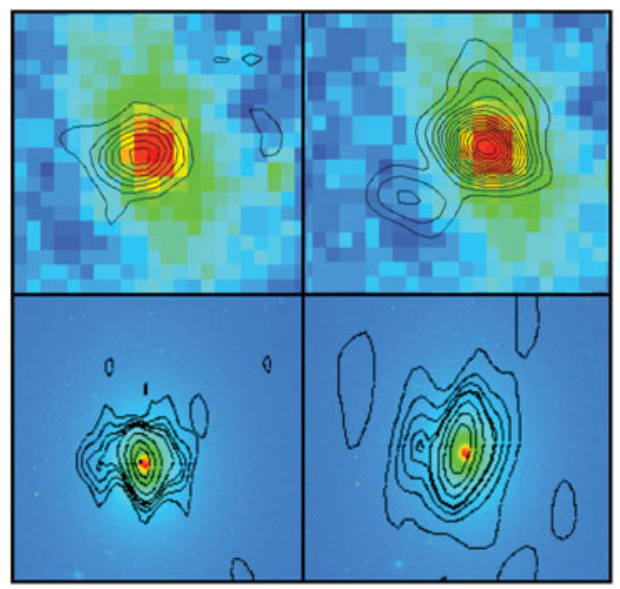

Figure 1. The $1.4 \mathrm{GHz}$ (top-left) and $610 \mathrm{MHz}$ (top-right) radio map of J1416, overlaid on DSS image of the central galaxy. The $1.4 \mathrm{GHz}$ (bottom-left) and $610 \mathrm{MHz}$ (bottom-right) radio map of ESO 3060170, overlaid on high resolution HST image of the central galaxy

\section{Estimate of Mechanical Heating}

The sample consists of 5 fossil systems, adopted from Khosroshahi, Ponman \& Jones 2007. In two cases, ESO 3060170 and J1416, we observe radio lobes extended to about 4 and $28 \mathrm{kpc}$ from the central source respectively. The lobes are detected clearly in 610 $\mathrm{MHz}$ for both sources but in $1.4 \mathrm{GHz}$, radio lobe is only visible for ESO 3060170 (Fig. 1). So the results corresponds to duty cycle of $40 \%(2 / 5)$ for AGN activity in galaxy groups.

We define two cooling radius within which the X-ray loss are determined. First the radius that cooling time falls below the Hubble time called $R_{c}$ and second the radius at which the temperature drops indicating cool core, $R_{c c}\left(R_{c c}<R_{c}\right)$. To calculate heating rate of radio bubbles, we adapted three different time scales introduced by Birzan et al. (2004) and the over pressure factors of 2 and 10 for the expanding bubble (Jetha et al. 2008). For ESO 3060170, mechanical power changes from 0.5 to $5.3 \times 10^{42} \mathrm{ergs}^{-1}$ applying different time scales and over pressure factors. Although this is quite sufficient to stop cooling within the $R_{c c}$ with X-ray loss of $\sim 10^{41} \mathrm{ergs}^{-1}$, it is not comparable to the Xray loss within $R_{c}$, with $L_{x} \sim 10^{43} \mathrm{ergs}^{-1}$. We obtained the same result for J1416. The mechanical luminosity of radio lobe is $0.2-1.2 \times 10^{43} \mathrm{ergs}^{-1}$, enough to quench cooling rate within $R_{c c}\left(L_{x}=2.8 \times 10^{42} \mathrm{ergs}^{-1}\right)$ but not sufficient to balance the cooling at higher radius $\left(R_{c}\right)$ with X-ray luminosity of $2.5 \times 10^{43} \mathrm{ergs}^{-1}$.

\section{Acknowledgments}

H.Miraghaei would like to thanks the National Elite Foundation, Iran, for partial financial support.

\section{References}

Bîrzan L., Rafferty D. A., McNamara B. R., Wise M. W. \& Nulsen P. E. J. 2004, ApJ, 607, 800 Jones L. R., Ponman T. J., Horton A., Babul A., Ebeling H. \& Burke D. J. 2003, MNRAS, 343, 627

Peterson J. R. \& Fabian A. C. 2006, Phys. Rep., 427, 1

Gitti M., Brighenti F. \& McNamara B. R. 2012, Adv. Astron., 2012, 1

Jetha N. N., Hardcastle M. J., Babul A., O'Sullivan E., Ponman T. J., Raychaudhury S., \& Vrtilek J. 2008, MNRAS, 384, 1344

Khosroshahi H. G., Ponman T. J., \& Jones L. R. 2007, MNRAS, 377, 595 